Journal of Mathematics and Statistics 8 (1): 15-23, 2012

ISSN 1549-3644

C 2012 Science Publications

\title{
Transient MHD Mixed Double Diffusive Convection Along a Vertical Plate Embedded in a Non-Darcy Porous Medium with Suction or Injection
}

\author{
Khaled K. Jaber \\ Department of Mathematics, \\ Faculty of Science and Information Technology, Zarqa University, Jordan
}

\begin{abstract}
Problem statement: Transient non-Darcy mixed double convection from a semi-infinite, isothermal vertical plate embedded in a homogeneous porous medium, in the presence of surface suction or injection had been numerically investigated. Approach: Forchheimer extension was considered in the flow equations. Appropriate transformations were employed to transform the derived partial differential equations governing the problem under consideration on the assumption of a small magnetic Reynolds number into a system of non linear ordinary differential equations, which were integrated by the fourth-order Runge-Kutta method. Results: Numerical results illustrating the effects of all involved parameters on the transient velocity, temperature and concentration profiles, the local Nussle, the local Sherwood numbers and the local skin fiction coefficient, were presented and discussed. The results were compared with those known from literature. Conclusion: Velocity and temperature increase due to the increasing of the parameters involved in the problem, while the increasing in the solutal dispersion parameter decreases the mass transfer coefficint.
\end{abstract}

Key words: Uniform magnetic, reynolds number, appropriate transformations, runge-kutta method, isothermal vertical, porous medium, transient non-darcy flow

\section{INTRODUCTION}

Convection heat transfer and flow through porous medium is phenomenon of great interest from both theoretical and practical point of view. This is because of its important applications in several geophysical, environmental and engineering fields. These applications include geothermal and petroleum resources, in-site combustion of oil shale, boiling enhancement using porous coatings, compact heat exchangers, packed bed reactors or absorbent, high performance of building insulation and others. Freeconvection flows past a semi-infinite vertical plate have been studied under different physical conditions by Irwan et al. (2010). Free-convection flow with mass transfer along a vertical plate in the presence of magnetic field has been investigated by Elbashbashy (1998). However, Aboeldabab and Elbarbary (2001) studied the Hall current effects on MHD free-convection flow past a semi-infinite vertical plate with mass transfer. Very recently, many investigations studying consequent flow and heat transfer characteristics that are brought about by the movement of a stretched permeable and impermeable. Isothermal and non isothermal surface with power law variation have been reported (see for instance, Abo-Eldahab and Gendy (2000) and AboEldahab et al. (2007). Most studies of natural convection in porous media have been based on Darcy's law which is applicable for slow flows and does not account for non-Darcian inertial effects. Nield and Bejan (2006) have made a comprehensive review of the growing volume of study devoted to heat transfer and flow through porous medium. Although, there is still a great deal of theoretical as well as practical interest in this area of research.

Most of the existing works on mixed convection flow over heated bodies embedded in fluid-saturated porous medium are concerned with steady state conditions (Merkin, 1980; Hsieh et al., 1993; Takhar and Beg, 1996). On the other hand, transient convection flow problems have not received as much attention. This is because that the transient heat transfer is usually difficult to solve either analytically or numerically. In fact there is no actual flow situation, which does not involve unsteadiness and examples of transient convective flows are numerous, for example, cooling of electronic devices in which the heat generation is not constant but time varying. Perhaps, the first study on transient boundary layer on flat plate was made by Johnson and Ping (1978). Raptis (1983) studied the case if two-dimensional free convection over a vertical plate embedded in a porous medium using the perturbation method. Al-Nimr and Masoud (1998) analyzed the problem of transient free convection flow 
over an impermeable vertical flat plate embedded in porous medium using Laplace transformation method. Extended Al-Nimr and Masoud (1998) study by considering the effect of variable suction on transient free convection. It is worth mentioning that all of these works the Ergun inertial effect was neglected. Also these studies were based on the assumption that the flow quantities are independent on the longitudinal direction (x-direction). This assumption leads to a constant free boundary layers thickness (momentum as well as thermal). Actually, it is impossible for the transient flow to be $\mathrm{x}$-independent. Shigeo (1989) solved the problem of transient forced in Darcy flow using the second-order upwind finite difference method. Nakayama and Ebinuma (1990) studied the inertial effects on transient forced convection for suddenly heated plate using the Frochheimer-extended Darcy model. Bejan and Nield (1991) obtained analytical solution for the three regimes, namely, the initial, transient period and steady heat transfer in a Darcian-porous medium. Analyzed the problem of transient free convection from suddenly cooled vertical in a Darcian-porous medium using asymptotic expansion method, while by Cheng and Pop (1983) used the integral method to study the same problem. Harris et al. (1996; 1997) and Ingham et al. (1982) have produced very detailed studies of the problem of transient free convection flow from vertical isothermal plate immersed in a fluid-saturated Darcian-porous medium when the temperature of the plate or the heat flux is suddenly changed. Harris et al. (1999) studied the problem of transient mixed convection flow from vertical isothermal plate immersed in a fluidsaturated Darcian-porous medium. Al-Odat (2004) extended Harris problem by considering the effect of variable suction on transient free-convection. Based on the above brief review, it is obvious that the transient non-Darcy MHD mixed convection flow along a vertical permeable flat plate embedded in porous medium has not been investigated yet. Therefore, the aim of the present study is to drop the steady state restriction and examine the time evolution of non-Darcy MHD mixed convection flow over a vertical isothermal semi-infinite flat plate immersed in a porous medium with wall suction or injection, when the plate temperature is suddenly raised from that of the ambient fluid. Appropriate transformations are employed to transform the partial differential equations, governing non-Darcy flow and heat transfer, to a non-similar form. The transformed equations have been solved numerically using the shooting technique with the fourth-order RungeKutta method.

\section{MATERIALS AND METHODS}

Mathematical formulation: Consider the unsteady, tow-dimensional, non-Darcy MHD mixed convection flow heat and mass transfer of laminar, incompressible and electrically conducting fluid over a semi-infinite vertical plate embedded in a porous medium with surface suction. A uniform magnetic field is applied normal to the plate. The schematic diagram of the physical problem is shown in Fig. 1. Initially the wall and the surrounding porous medium are at uniform and constant temperature $\mathrm{T}_{\infty}$, constant concentration $\mathrm{C}_{\infty}$ and constant velocity $\mathrm{u}_{\infty}$ vertically past the plate inside the porous medium. At time $\mathrm{t}=0$, the vertical plate is suddenly heated isothermally with imposed suction which in turn, suddenly raised the temperature and concentration at the wall to $\mathrm{T}_{\mathrm{w}}$ and $\mathrm{C}_{\mathrm{w}}$, respectively and they maintained at these values for $t>0$. It is assumed that the fluid properties are constant except the influence of density variation with temperature, which is considered only in the body force term of the momentum equation. Both the fluid and solid matrix are assumed to be in local thermal equilibrium. Under these assumptions along with Boussinesq approximation, the transient laminar boundary layer Eq. 1-4 are given by:

$$
\begin{aligned}
& \frac{\partial u}{\partial x}+\frac{\partial v}{\partial y}=0 \\
& \frac{\partial u}{\partial y}+\frac{F \sqrt{K}}{v} \frac{\partial u^{2}}{\partial y}= \pm \frac{K g \beta_{T}}{v} \frac{\partial T}{\partial y}-\frac{\sigma B_{0}^{2} K}{\rho v} \frac{\partial u}{\partial y}+\frac{K g \beta_{C}}{v} \frac{\partial C}{\partial y} \\
& \frac{\partial T}{\partial t}+u \frac{\partial T}{\partial x}+v \frac{\partial T}{\partial y}=\frac{\partial}{\partial y}\left(\alpha_{e} \frac{\partial T}{\partial y}\right) \\
& \frac{\partial C}{\partial t}+u \frac{\partial C}{\partial x}+v \frac{\partial C}{\partial y}=\frac{\partial}{\partial y}\left(D_{e} \frac{\partial C}{\partial y}\right)
\end{aligned}
$$
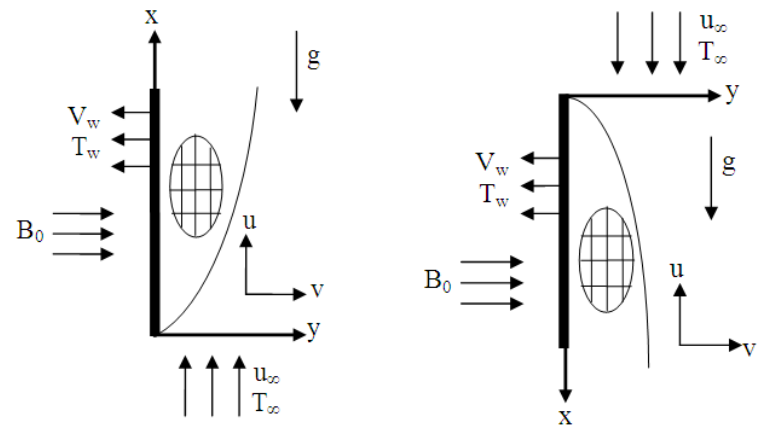

Fig. 1: Schematic diagram for the problem 
The appropriate boundary conditions relevant to the problem are Eq. 5:

$\mathrm{V}(\mathrm{x}, 0, \mathrm{t})=-\mathrm{V}_{\mathrm{w}}(\mathrm{x}) \mathrm{T}(\mathrm{x}, 0, \mathrm{t})=\mathrm{T}_{\mathrm{w}}, \mathrm{C}(\mathrm{x}, 0, \mathrm{t})=\mathrm{C}_{\mathrm{w}}$

$\mathrm{U}(\mathrm{x}, \infty, \mathrm{t})=\mathrm{U} \infty \mathrm{T}(\mathrm{w}, \infty, \mathrm{t})=\mathrm{T} \infty, \mathrm{C}(\mathrm{x}, \infty, \mathrm{t})=\mathrm{C} \infty$

And the initial conditions are Eq. 6:

$\mathrm{U}(\mathrm{x}, \mathrm{y}, 0)=\mathrm{v}(\mathrm{x}, \mathrm{y}, 0)=, 0$,

$\mathrm{T}(\mathrm{x}, \mathrm{y}, 0)=\mathrm{T} \infty, \mathrm{C}(\mathrm{x}, \mathrm{y}, 0)=\mathrm{C} \infty$

Where:

$(\mathrm{x}, \mathrm{y}) \quad=$ The dimensional distance along and normal to the plate

$(\mathrm{u}, \mathrm{v}) \quad=$ The velocity components in $\mathrm{x}$-and $\mathrm{y}$ direction

$\mathrm{T}=$ The temperature

$\mathrm{C}=$ The concentration

$\beta=$ The coefficient of thermal expansion

$\mathrm{v}=\quad=$ The effective kinematics viscosity

$\mathrm{F} \quad=$ The dimensional inertial coefficient

$\mathrm{K}=$ The permeability of the medium

$\mathrm{g} \quad=$ The gravitational acceleration

$\sigma=$ The heat capacity

$\rho \quad=$ The fluid density

$\alpha_{e}$ and $D_{e}=$ The effective thermal and solutal diffusivities

Following to Telles and Travisan (1993) the expression for $\alpha_{\mathrm{e}}$ vand $\mathrm{D}_{\mathrm{e}}$ can be written as $\alpha \mathrm{e}=\alpha+$ $\chi$ ud and $\mathrm{D}_{\mathrm{e}}=\mathrm{D}+$ gud and where $\alpha$ and $\mathrm{D}$ are the molecular thermal and solutal diffusivities, respectively, whereas $\chi$ ud and $\varsigma$ ud represent the effective thermal and soluta 1 diffusivities due to the transverse thermal and solutal dispersion, where $\chi$ and $\varsigma$ are coefficient of dispersion thermal and solutal diffusivities and $d$ is the pore diameter. The plus sign is to designate the flow as an aiding flow when the buoyancy force has a component in the direction of the free stream and the minus sign as an opposing flow when the buoyancy component is opposite to the free stream velocity. The subscripts $\mathrm{w}$ and $\infty$ indicate the conditions at the wall and at the outer edge of the boundary layer, respectively.

In order to reduce the number of independent variables from three to two and to make the governing equations dimensionless, the following transformation is applied:

$$
\eta=y\left[\frac{u_{\infty}}{2 \alpha x}\right]^{1 / 2} \xi^{-1 / 2} \psi=\left(2 \alpha u_{\infty} x\right)^{1 / 2} \xi^{1 / 2} f(\xi, \eta) \tau=\frac{u_{\infty} t}{x}
$$

$$
\xi=1-\mathrm{e}^{-\tau} \theta=\frac{\mathrm{T}-\mathrm{T}_{\infty}}{\mathrm{T}_{\mathrm{w}}-\mathrm{T}_{\infty}} \mathrm{u}=\frac{\partial \psi}{\partial \mathrm{y}} \mathrm{v}=-\frac{\partial \psi}{\partial \mathrm{x}}
$$

Using the above transformation in Eq. 1-4, we find that Eq. 1 is identically satisfied and Eq. 2-4 are reduced to Eq. 7-9:

$$
\begin{aligned}
& \mathrm{f}^{\prime \prime}+2 \Gamma \mathrm{f}^{\prime} \mathrm{f}^{\prime}= \pm \lambda \theta^{\prime}-\mathrm{Ha}^{2} \mathrm{f}^{\prime \prime}+\lambda \mathrm{N} \varphi^{\prime} \\
& \theta^{\prime \prime}+\delta_{\mathrm{t}}\left(\mathrm{f}^{\prime} \theta^{\prime \prime}+\mathrm{f}^{\prime \prime} \theta^{\prime}\right)+[\xi+(1-\xi) \ln (1-\xi)] \\
& \mathrm{f}^{\prime}+2 \xi(1-\xi) \ln (1-\xi) \theta^{\prime} \frac{\partial \mathrm{f}}{\partial \xi} \\
& =2 \xi(1-\xi)\left[1+\ln (1-\xi) \mathrm{f}^{\prime}\right] \frac{\partial \theta}{\partial \xi} \\
& \varphi^{\prime \prime}+\delta_{\mathrm{s}}\left(\mathrm{f}^{\prime} \varphi^{\prime \prime}+\mathrm{f}^{\prime \prime} \varphi^{\prime}\right)+\operatorname{Le}[\xi+(1-\xi) \ln (1-\xi)] \\
& \varphi^{\prime} \mathrm{f}+2 \operatorname{Le} \xi(1-\xi) \ln (1-\xi) \varphi^{\prime} \frac{\partial \mathrm{f}}{\partial \xi} \\
& =2 \operatorname{Le} \xi(1-\xi)\left[1+\ln (1-\xi) \mathrm{f}^{\prime}\right] \frac{\partial \varphi}{\partial \xi}
\end{aligned}
$$

where, the prime denotes partial differentiation with respect to $\eta$. The boundary conditions (Abo-Eldahab and Gendy, 2000) are reduced to Eq. 10:

at $=0: \mathrm{f}(\xi, 0)=\mathrm{f}_{\mathrm{w}}, \theta(\xi, 0)=1, \phi(\xi, 0)=1$

at $\longrightarrow \infty$ : f' $(\xi, \infty)=1, \theta(\xi, \infty)=0, \phi(\xi, \infty)=0$

And the initial conditions (6) are reduced to Eq. 11:

$f(0 \eta)=\theta(0 \eta)=\phi(0, \eta)=0$

Where:

$$
\begin{aligned}
& \lambda=\frac{\operatorname{Kg} \beta\left(\mathrm{T}_{\mathrm{w}}-\mathrm{T}_{\infty}\right)}{v \mathrm{u}_{\infty}}=\frac{\mathrm{Ra}}{\mathrm{Pe}}, \mathrm{Re}=\frac{\mathrm{u}_{\infty} \mathrm{x}}{\mathrm{v}}, \\
& \mathrm{Pe}=\frac{\mathrm{u}_{\infty} \mathrm{x}}{\alpha}, \mathrm{Ra}=\frac{\mathrm{Kg} \beta\left(\mathrm{T}_{\mathrm{w}}-\mathrm{T}_{\infty}\right) \mathrm{x}}{\alpha v}, \\
& \mathrm{f}_{\mathrm{w}}=\mathrm{V}_{\mathrm{w}}(\mathrm{x}) \sqrt{\frac{\mathrm{x}}{\alpha \mathrm{u}_{\infty}}}, \Gamma=\frac{\mathrm{F} \sqrt{\mathrm{Ku}_{\infty}}}{v}, \\
& \mathrm{Le}=\frac{\alpha}{\mathrm{D}}, \mathrm{v}, \quad \delta_{\mathrm{t}}=\frac{\chi \mathrm{u}_{\infty} \mathrm{d}}{\alpha} \delta_{\mathrm{t}}=\delta_{\mathrm{s}}=\frac{\zeta \mathrm{u}_{\infty} \mathrm{d}}{\mathrm{D}},
\end{aligned}
$$

Are the mixed convection parameter, Peclet number, Reynolds number, Raleigh number, the dimensionless wall mass transfer parameter, the dimensionless inertial parameter, Lewis number, 
buoyancy ratio parameter, thermal dispersion parameter and solutal dispersion parameter, respectively. It should be noted that $\lambda=0$ corresponds to the pure forced convection regime, while large values of $\lambda$ corresponds to the free convection regime dominate. Also, the normal velocity at the surface must be inversely proportional to the square root of $x$, in order to make $f_{w}$ independent of $\mathrm{x}$.

The physical quantities of fundamental interest of heat and mass transfer are the local friction factor, the local heat transfer and the local mass transfer coefficients in terms of the local skin friction coefficient, the local Nusselt and Sherwood numbers, respectively. The local skin friction coefficient can be expressed as Eq. 12:

$$
\mathrm{C}_{\mathrm{f}}=\frac{2 \mu}{\rho \mathrm{u}_{\infty}^{2}}\left(\frac{\partial \mathrm{u}}{\partial \mathrm{y}}\right)_{\mathrm{y}=0}=\sqrt{2 \mathrm{Pe}} \operatorname{Re}^{-1} \xi^{-1 / 2} \mathrm{f}^{\prime \prime}(\xi, 0)
$$

Similarly, the Nusselt number can be expressed as Eq. 13:

$$
\mathrm{Nu}=\frac{\mathrm{x}}{\left(\mathrm{T}_{\mathrm{w}}-\mathrm{T}_{\infty}\right)}\left(\frac{\partial \mathrm{T}}{\partial \mathrm{y}}\right)_{\mathrm{y}=0}=\frac{1}{2} \sqrt{2 \mathrm{Pe}} \xi^{-1 / 2} \theta^{\prime}(\xi, 0)
$$

Also, the Sherwood number can be written as Eq. 14:

$$
\mathrm{Sh}=\frac{\mathrm{x}}{\left(\mathrm{C}_{\mathrm{w}}-\mathrm{C}_{\infty}\right)}\left(\frac{\partial \mathrm{C}}{\partial \mathrm{y}}\right)_{\mathrm{y}=0}=\frac{1}{2} \sqrt{2 \mathrm{Pe}} \xi^{-1 / 2} \varphi^{\prime}(\xi, 0)
$$

\section{RESULTS AND DISCUSSION}

The influence of various parameters on the transient behavior of the laminar mixed convection flow heat and mass transfer along an isothermal vertical plate embedded in a porous medium is examined and discussed. During the early stages, heat and mass are transferred from the surface to the surrounding fluid in a one-dimension conduction process, similar to heat conduction in a semi-infinite conducting medium. After the passage of this stage, the transient process becomes and remains two-dimensioned until steady state conditions are reached.

Before solving the system of equations (Nield and Bejan, 2006; Merkin, 1980; Hsieh et al., 1993) with the boundary conditions (Takhar and Beg, 1996) and initial conditions (Johnson and Ping, 1978) it can be noticed that the equation (Hsieh et al., 1993) is uncoupled with any of the momentum or energy equation, also Hartmann Ha, inertial $\Gamma$ and the mixed convection $\chi$ parameters do not affect the concentration and slightly affect the temperature so no figure of these variables is presented herein.

Figure 2 shows the influence of the Hartmann number on the velocity profiles. We observe that the fluid velocity decrease owing to the increase of $\mathrm{Ha}$. This is because the application of a transverse magnetic field to an electrically conducting fluid gives rise to a resistive force which has a tendency to slow down the motion of the fluid in the boundary layer. Figure 3 and 4 shows the effect of the inertial parameter on the transient velocity profiles within the boundary layer for aiding and opposing flow, respectively.

As shown in Fig. 3 when the buoyancy is aiding the flow, the axial velocity evolves from non-zero wall velocity to uniform free stream for all values of time.

It is clear that (for aiding as well as opposing flow), the velocity profiles predicted by the non-Darcy model differ significantly from those obtained based on the Darcy model.

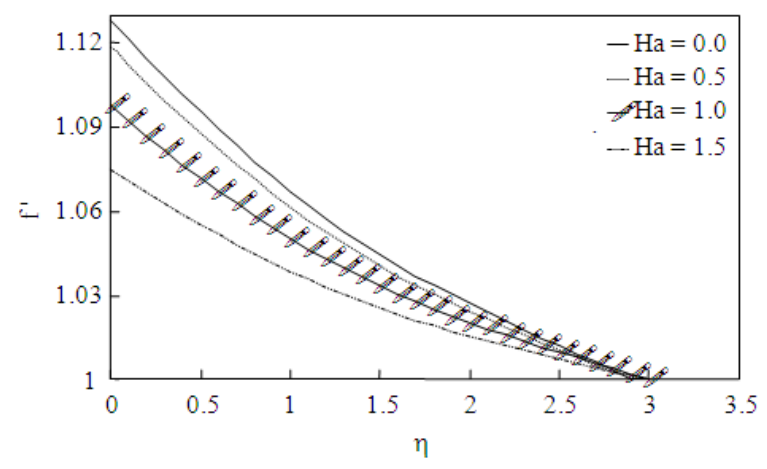

Fig. 2: Variation of the transient velocity profiles with the Hartmann number $\mathrm{Ha}$ for $\mathrm{N}=\Gamma=1$, Le $=$ $0.5, \xi=\lambda=\delta 1=\delta 2=0.2, \mathrm{f}_{\mathrm{w}}=0$

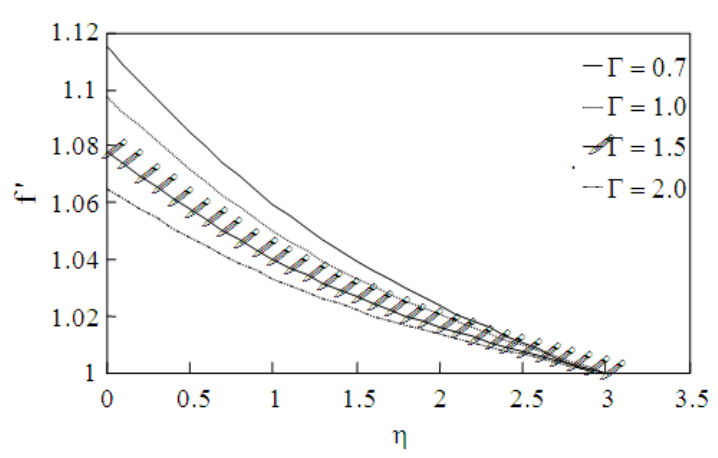

Fig. 3: Variation of the transient velocity profiles for aidding flow with the inertial parameter $\Gamma$ for $\mathrm{N}$ $=\mathrm{Ha}=1, \mathrm{Le}=0.5, \xi=\lambda=\delta 1=\delta=0.2, \mathrm{f}_{\mathrm{w}}=0$ 


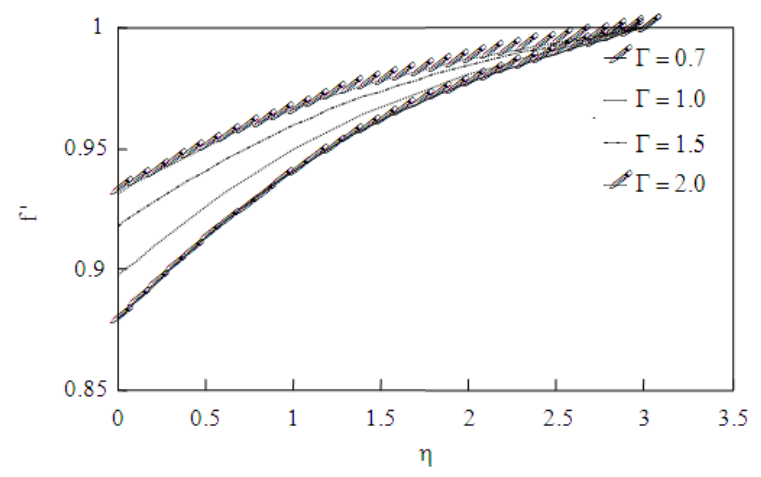

Fig. 4: Variation of the transient velocity profiles for opposing flow with the inertial parameter $\Gamma$ for $\mathrm{N}=\mathrm{Ha}=1, \mathrm{Le}=0.5, \xi=\delta 1=\delta=0.2, \mathrm{f}_{\mathrm{w}}=0, \lambda$ $=-0.02$

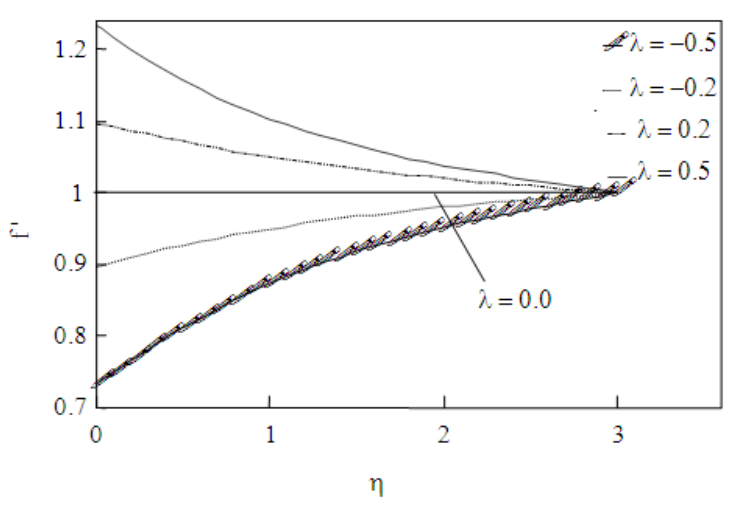

Fig. 5: Variation of the transient velocity profiles with the mixed convection parameter $\lambda$ for $\mathrm{N}=\mathrm{Ha}=$ $\Gamma=1, \mathrm{Le}=0.5, \xi=\delta 1=\delta 2=0.2, \mathrm{f}_{\mathrm{w}}=0$

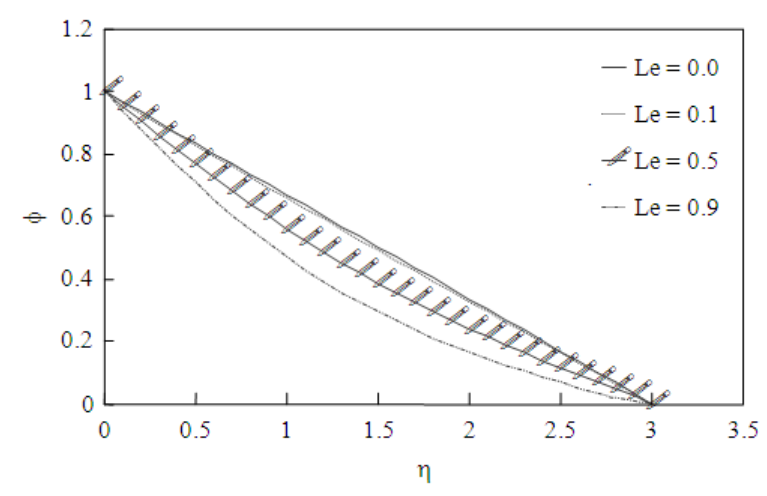

Fig. 6: Variation of the transient concentration profiles with the Lewis number Le for $\mathrm{N}=\mathrm{Ha}$ $=\Gamma=1, \xi, \lambda=\delta_{1}=\delta_{2}=0.2, \mathrm{f}_{\mathrm{w}}=0$
The inertia effect tends to decrease the velocity, since the fluid inertia provides an additional pressure loss in the flow field. Also, the hydrodynamic boundary layers predicted by the Darcy model are thicker than those predicted by the non-Darcy model. The influence of mixed convection parameter on the transient non-Darcy velocity profiles for aiding and opposing flow is shown in Fig. 5.

It is obvious that the velocity increases as the mixed convection parameter increase. Figure 6 illustrates the effect of the Lewis number Le on the concentration profiles. It is obvious that the increase in the Lewis number Le tends to decrease the concentration profiles.

The effect of time $\xi$ for aiding flow case $\chi=0.2$ on the velocity, temperature and concentration profiles are shown in Fig.7-9 it is obvious that the flow velocity, temperature and concentration profiles increasing with time progressing. As $\xi \rightarrow 1(\tau \rightarrow \infty)$ the fluid velocity, temperature and concentration distributions approach steady state conditions. The influence of the dimensionless wall mass transfer parameter $f_{w}$ on the temperature profiles is shown in Fig. 10. It is noted that as $f_{w}$ increases the temperature profiles increase. The value of $f_{w}=0$ corresponds to an impermeable surface. The increase of the buoyancy ratio parameter increases the velocity profiles in the flow boundary layer as shown in Fig. 11. Figure 12 and 13 present typical profiles of fluid velocity and temperature for different values of the thermal dispersion parameter $\delta_{t}$. It is observed that the temperature rises more quickly than the velocity distribution and the thermal boundary layer becomes thicker as the thermal dispersion thicker as the

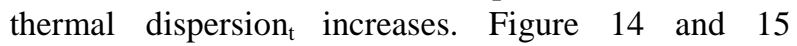
elucidate the influence of the solutal dispersion parameter $\delta_{\mathrm{s}}$ on the velocity and concentration profiles.

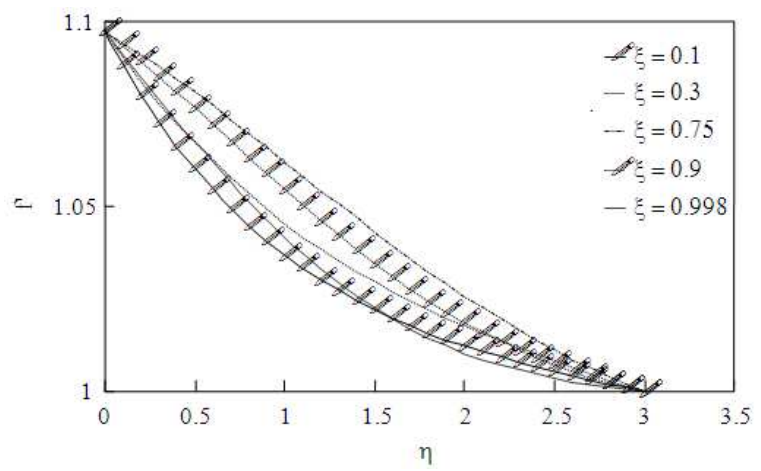

Fig. 7: Variation of the transient velocity profiles with the time $\xi$ for $\mathrm{N}=\mathrm{Ha}=\Gamma=1, \mathrm{Le}=0.5, \delta 1=$ $\delta 2=0.2, f_{w}=0$ 


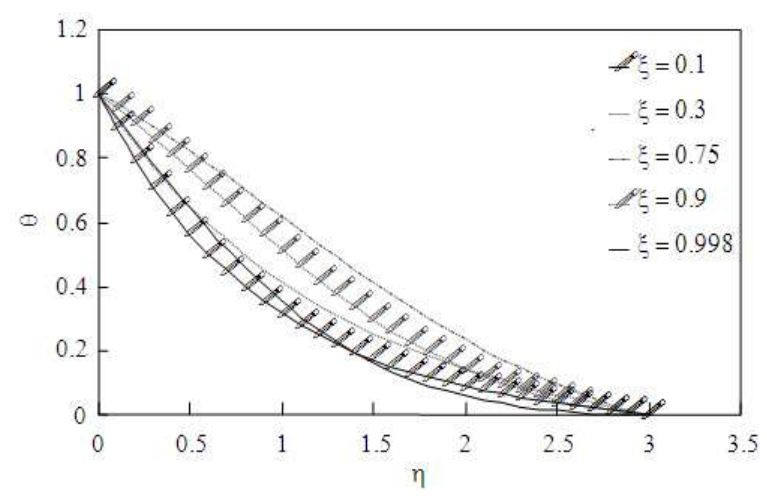

Fig. 8: Variation of the transient temperaure profiles with the time $\xi$ for $\mathrm{N}=\mathrm{Ha}=\Gamma=1, \mathrm{Le}=0.5, \delta 1$ $=\delta_{2}=0.2, \mathrm{f}_{\mathrm{w}}=0$

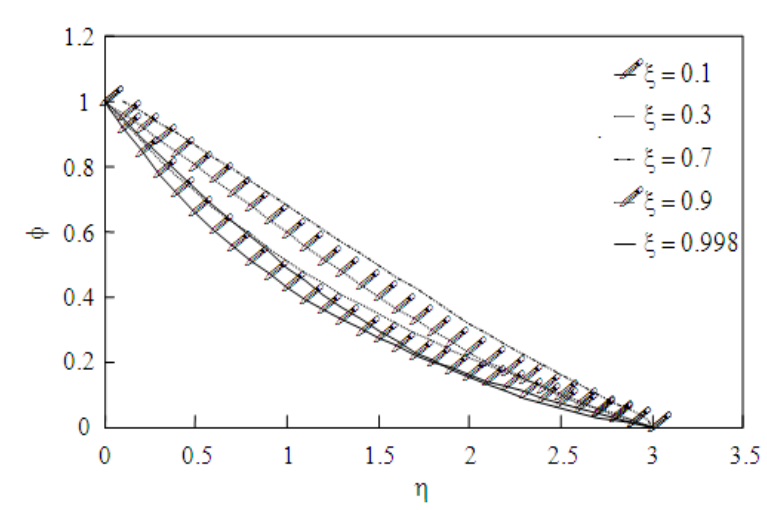

Fig. 9: Variation of the transient concentration profiles with the time $\xi$ for $\mathrm{N}=\mathrm{Ha}=\Gamma=1$, Le $=$ $0.5, \delta 1=\delta 2=0.2, \mathrm{f}_{\mathrm{w}}=0$

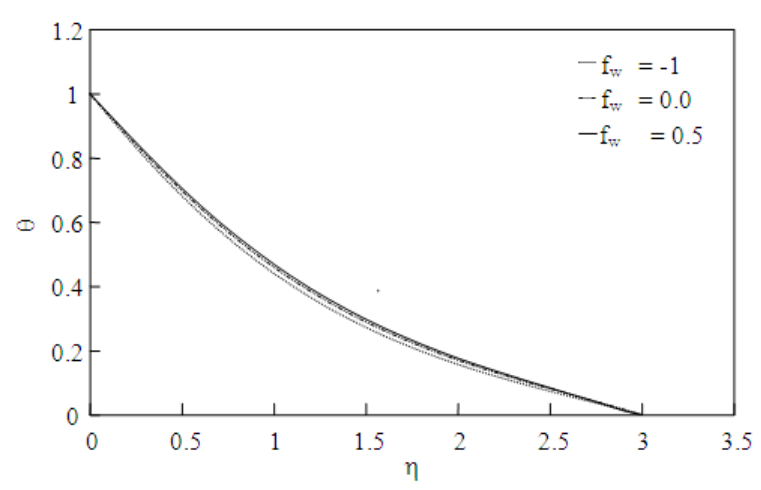

Fig.10: Variation of the transient temperature profiles with $\mathrm{f}_{\mathrm{w}}$ for $\mathrm{N}=\mathrm{Ha}=\Gamma=1, \xi=\lambda=\delta_{1}=\delta_{2} 0.2$, Le $=0.5$

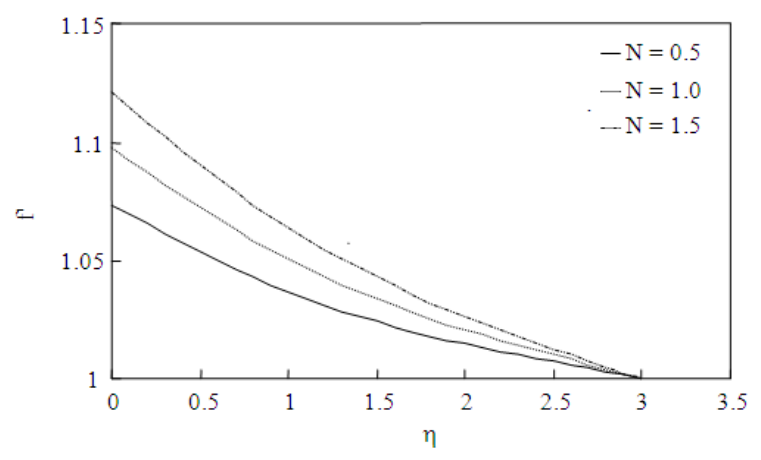

Fig. 11: Variation of the transient velocity profiles with the buoyancy ratio parameter $\mathrm{N}$ for $\mathrm{Ha}=\Gamma=$ $1, \xi=\lambda=\delta 1=\delta 2=0.2 \mathrm{Le}=0.5, \mathrm{f}_{\mathrm{w}}=0$

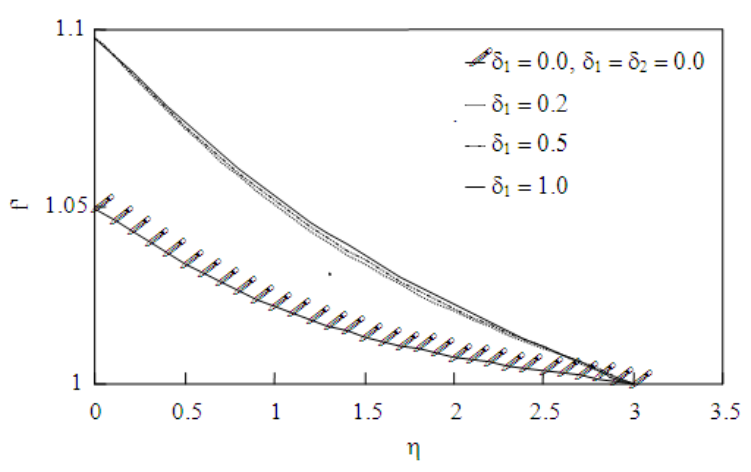

Fig. 12: Variation of the transient velocity profiles with the dispertion thermal diffusivity parameter $\delta_{1}$ for $\mathrm{N}=\mathrm{Ha}=\Gamma=1, \xi=\chi=\delta_{2}=$ $0.2, \mathrm{Le}=0.5, \mathrm{f}_{\mathrm{w}}=0$

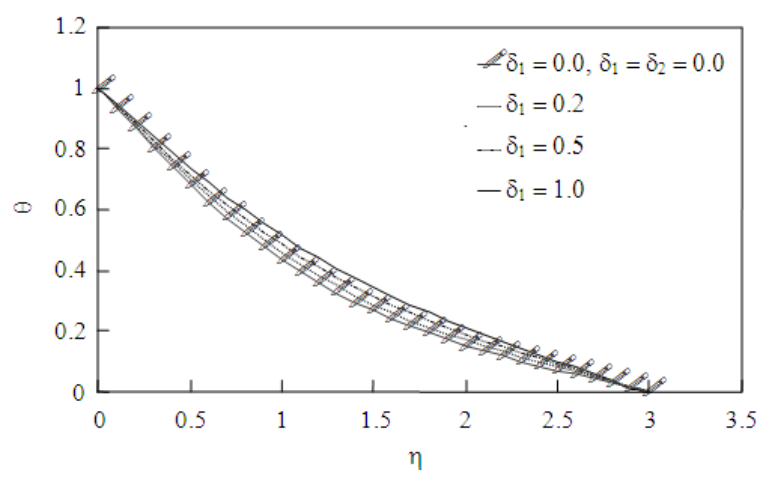

Fig. 13: Variation of the transient temperature profiles with the dispertion thermal diffusivity parameter $\delta_{1}$ for $\mathrm{N}=\mathrm{Ha}=\Gamma=1, \xi=\lambda=\delta_{2}=$ $\mathrm{Le}=0.5, \mathrm{f}_{\mathrm{w}}=0$ 
Table 1: Comparison of the values of $\mathrm{f}^{\prime}(\xi, 0)$ for various values of $\lambda \lambda$ Harris et al. (1999) Al-Odat (2004) Present study

\begin{tabular}{llll}
\hline-1 & 0.0 & 0.0001 & 0.00000 \\
0 & 1.0 & 1.0000 & 1.00000 \\
1 & 2.0 & 1.9998 & 2.00018 \\
2 & 3.0 & 2.9992 & 3.00016 \\
\hline
\end{tabular}

Table 2: Variation of $\theta(\xi, 0)$ and $\phi(\xi, 0)$ at the plate surface with Le, $\mathrm{f}_{\mathrm{w}} \delta, \delta$. and $\xi$ for $\mathrm{Ha}=\mathrm{N}=\Gamma=1.0$ and $\lambda=0.2$

\begin{tabular}{lclllcr}
\hline Le & \multicolumn{1}{c}{$\mathrm{f}_{\mathrm{w}}$} & $\delta_{\mathrm{t}}$ & $\delta_{\mathrm{s}}$ & $\xi$ & $-\theta^{\prime}(\xi, 0)$ & $-\phi^{\prime}(\xi, 0)$ \\
\hline 0.0 & 0.0 & 0.2 & 0.2 & 0.200 & 0.6368 & 0.3303 \\
0.1 & 0.0 & 0.2 & 0.2 & 0.200 & 0.6369 & 0.3432 \\
0.5 & 0.0 & 0.2 & 0.2 & 0.200 & 0.6371 & 0.4742 \\
0.9 & 0.0 & 0.2 & 0.2 & 0.200 & 0.6377 & 0.6088 \\
0.5 & -1.0 & 0.2 & 0.2 & 0.200 & 0.6926 & 0.4707 \\
0.5 & 0.2 & 0.2 & 0.2 & 0.200 & 0.6370 & 0.4742 \\
0.5 & 0.5 & 0.2 & 0.2 & 0.200 & 0.6103 & 0.4767 \\
0.5 & 0.0 & 0.5 & 0.2 & 0.200 & 0.5973 & 0.4742 \\
0.5 & 0.0 & 1.0 & 0.2 & 0.200 & 0.5503 & 0.4742 \\
0.5 & 0.0 & 0.2 & 1.0 & 0.200 & 0.6369 & 0.3954 \\
0.5 & 0.0 & 0.2 & 5.0 & 0.200 & 0.6368 & 0.3311 \\
0.5 & 0.0 & 0.2 & 0.2 & 0.100 & 1.1427 & 0.8186 \\
0.5 & 0.0 & 0.2 & 0.2 & 0.750 & 0.3104 & 0.3150 \\
0.5 & 0.0 & 0.2 & 0.2 & 0.900 & 0.4270 & 0.3672 \\
0.5 & 0.0 & 0.2 & 0.2 & 0.998 & 0.7314 & 0.5400 \\
\hline
\end{tabular}

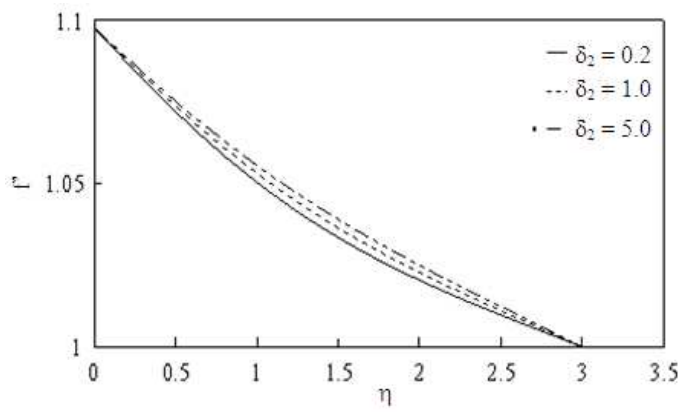

Fig. 14: Variation of the transient velocity profiles with the dispertion solutal diffusivity parameter $\delta_{2}$ for $\mathrm{N}=\mathrm{Ha}=\Gamma=1, \xi=\lambda=$ $\delta_{1}=0.2$, Le $=0.5, f_{w}=0$

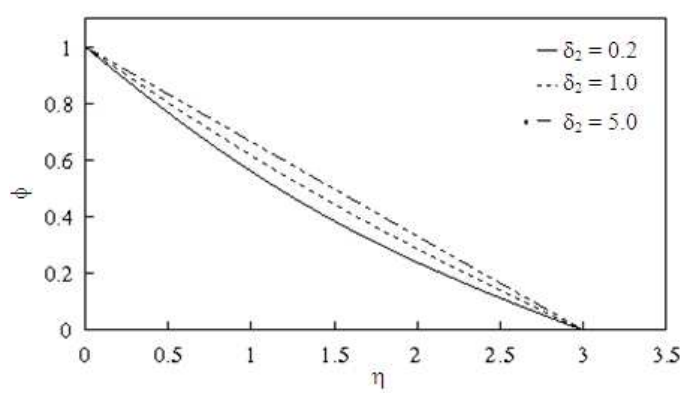

Fig. 15: Variation of the transient cocentration profiles with the dispertion solutal diffusivity parameter $\delta_{2}$ for $\mathrm{N}=\mathrm{Ha}=\Gamma=1, \xi=\lambda=$ $\delta_{1}=$ Le $=0.5, \mathrm{f}_{\mathrm{w}}=0$
Table 3: Variation of $\mathrm{f}^{\prime}(\xi, 0), \theta(\xi 0)$ and $\phi(\xi, 0)$ at the plate surface with $\mathrm{Ha}, \theta(\xi, 0)$ and $\phi$ for $\mathrm{Le}=0.5, \mathrm{f}_{\mathrm{w}}=0.0, \delta=\delta=\xi=0.2$

\begin{tabular}{lllllrc}
\hline $\mathrm{Ha}$ & $\Gamma$ & \multicolumn{1}{c}{$\lambda$} & $\mathrm{N}$ & $\mathrm{f}^{\prime \prime}(\xi, 0)$ & \multicolumn{1}{c}{$\theta^{\prime}(\xi, 0)$} & \multicolumn{1}{c}{$\phi^{\prime}(\xi, 0)$} \\
\hline 0 & 1.0 & 0.2 & 1.0 & 1.1283 & 0.6340 & 0.4742 \\
0.5 & 1.0 & 0.2 & 1.0 & 1.1188 & 0.6351 & 0.4742 \\
1.0 & 1.0 & 0.2 & 1.0 & 1.0976 & 0.6370 & 0.4742 \\
1.5 & 1.0 & 0.2 & 1.0 & 1.0751 & 0.6396 & 0.4742 \\
1.0 & 0.7 & 0.2 & 1.0 & 1.1154 & 0.6355 & 0.4752 \\
1.0 & 1.5 & 0.2 & 1.0 & 1.0782 & 0.6392 & 0.4758 \\
1.0 & 2.0 & 0.2 & 1.0 & 1.0653 & 0.6406 & 0.4769 \\
1.0 & 1.0 & -0.5 & 1.0 & 0.7321 & 0.6765 & 0.5057 \\
1.0 & 1.0 & -0.2 & 1.0 & 0.8974 & 0.6588 & 0.4911 \\
1.0 & 1.0 & 0.1 & 1.0 & 1.0494 & 0.6423 & 0.4782 \\
1.0 & 1.0 & 0.2 & 0.5 & 1.0736 & 0.6398 & 0.4762 \\
1.0 & 1.0 & 0.2 & 1.5 & 1.1209 & 0.6344 & 0.4723 \\
\hline
\end{tabular}

The increase in the values of $\delta_{\mathrm{s}}$ tends to increase the momentum and concentration boundary layers, which in turn increasing the velocity and concentration. It is worth mentioning that the concentration field is slightly responsive to the changes in the thermal dispersion $\delta_{t}$, also the increase in the solutal dispersion parameter $\delta_{\mathrm{s}}$ very slightly reduces the temperature profiles, so no figure of these variables is presented herein.

Table 1 shows a comparison of the present results for the skin friction $\mathrm{f}^{\prime}$ ' $(\xi, 0)$ with those reported by Harris et al. (1999) and Al-Odat (2004) for $\Gamma=\mathrm{Ha}=\mathrm{Le}$ $=0$ and various values of the mixed convection parameter $\lambda$, the results are found to be in good agreement. Our numerical values for f', $(\xi, 0) \theta$ ' $(\xi, 0)$ and $\phi^{\prime}(\xi, 0)$ are listed in Table 2 and 3. It is clear that the skin friction decreases owing to the increase of $\Gamma$ or $\mathrm{Ha}$ and increases owing to the increase of $\lambda$ while it does not affected by the variation of Le and $f_{w}$. The rate of heat and mass transfer increase as $\Gamma$, Ha or $\mathrm{f}_{\mathrm{w}}$ increases while they decrease as $\lambda, \xi$ increase. Finally the increase of Le increases the rate of mass transfer.

\section{CONCLUSION}

Transient non-Darcy MHD mixed double diffusive convection flow of an electrically conducting fluid over an isothermal semi-infinite, vertical plate embedded in a homogeneous porous medium, in the presence of a transverse uniform magnetic field and surface suction or injection has been numerically investigated. The non-Darcy model, which includes the Forchhimer extension, is employed to describe the flow in the porous medium. The plate is suddenly heated and its temperature and concentration are raised from $T_{\infty}$ to $T_{w}$ and from $\mathrm{C}_{\infty}$ to $\mathrm{C}_{\mathrm{w}}$, respectively. The governing partial differential equations are transformed to a non-similar form by introducing appropriate transformation. The transformed equations have been solved numerically using the fourth order Runge-Kutta method with the 
shooting technique. The effects of all involved parameters on the transient velocity, temperature, concentration profiles, the local skin friction coefficient, Nusselt number and Sherwood number are presented and discussed. It has been found that:

- Increasing the inertial parameter $\Gamma$ can cause a slight increase in the fluid temperature, mass concentration, the local Nusselt and Sherwood numbers and a significant decrease in fluid velocity and the local skin friction coefficient. Furthermore, it is worth mentioning that the inertial effect grows with time

- Increasing the mixed convection parameter $\lambda$ causes an increase in the fluid velocity and skin friction coefficient, also it reduce the heat and mass transfer coefficients for aiding flow, while an apposite trend is observed for the opposing flow case

- Increasing the Hartmann number $\mathrm{Ha}$ causes decrease in the fluid velocity and skin friction coefficient while it increases the heat and mass transfer coefficients

- Increasing the Lewis number Le can cause a decrease in mass concentration and increase the mass transfer coefficient

- The fluid velocity, fluid temperature, mass concentration, heat and mass transfer coefficients increase as the time $\xi$ increases from zero to 0.75 then they decrease to reach the steady state as $\xi-1$ $(\tau \rightarrow \infty)$

- Increasing the buoyancy ratio parameter increases the fluid velocity and skin friction while it decreases both the heat and mass transfer coefficients

- Increasing the thermal dispersion parameter increases both the velocity and fluid temperature while it decreases the heat transfer coefficient

- Increasing the solutal dispersion parameter increases both the velocity and fluid temperature while it decreases the mass transfer coefficient

- Finally, increasing $f_{w}$ increases the mass transfer and decreases the heat transfer coefficients

\section{REFERENCES}

Aboeldabab, E.M. and E.M.E. Elbarbary, 2001. Hall current effect on magnetohydrodynamic freeconvection flow past a semi-infinite vertical plate with mass transfer. Int. J Eng. Sci., 39: 1641-1652. DOI: 10.1016/S0020-7225(01)00020-9
Abo-Eldahab, E.M., M.A. El-Aziz, A.M. Salem and K.K. Jaber, 2007. Hall current effect on MHD mixed convection flow from an inclined continuously stretching surface with blowing/suction and internal heat generation/absorption. Applied Math. Modell., 31: 1829-1846. DOI: 10.1016/j.apm.2006.06.017

Abo-Eldahab, E.M. and M.S.E. Gendy, 2000. Radiation effect on convective heat transfer in an electrically conducting fluid at a stretching surface with variable viscosity and uniform free stream. Phys. Scripta, 62: 321-321. DOI: 10.1238/Physica.Regular.062a00321

Al-Nimr, M.A. and S. Masoud, 1998. Unsteady free convection flow over a vertical flat plate immersed in a porous medium. Fluid Dyn. Res., 23: 153-153. DOI: 10.1016/S0169-5983(97)00059-2

Al-Odat, M.Q., 2004. Transient non-Darcy mixed convection along a vertical surface in porous medium with suction or injection. Applied Math. Comput., 156: 679-694. DOI: 10.1016/j.amc.2003.08.017

Bejan, A. and D.A. Nield, 1991. Transient forced convection near a suddenly heated plate in a porous medium. Int. Commun. Heat Mass Transfer, 18: 83-91. DOI: 10.1016/07351933(91)90010-2

Cheng, P., and I. Pop, 1983. Transient free convection about a vertical flat plate embedded in a porous medium. International Journal of Engineering Science, 22: 253-264. doi:10.1016/00207225(84)90006-5

Elbashbashy, E.M.A., 1998. Heat transfer over a stretching surface with variable surface heat flux. J. Phys. D: Applied Phys., 31: 1951-1951. DOI: 10.1088/00223727/31/16/002

Harris, S.D., D.B. Ingham and I. Pop, 1996. Transient free convection from a vertical plate subjected to a change in surface heat flux in porous media. Fluid Dyn. Res., 18: 313-313. DOI: 10.1016/01695983(96)00025-1

Harris, S.D., D.B. Ingham and I. Pop, 1997. Free convection from a vertical plate in a porous medium subjected to a porous medium subjected to a sudden change in surface heat flux. Transport Porous Media, 26: 207-226. DOI: 10.1023/A:1006550510374

Harris, S.D., D.B. Inghamx and I. Pop, 1999. Unsteady mixed convection boundary-layer flow on a vertical surface in a porous medium. Int. J. Heat Mass Transfer, 42: 357-372. DOI: 10.1016/S00179310(98)00209-9 
Hsieh, J.C., T.S. Chen and B.F. Armaly, 1993. Nonsimilarity solutions for mixed convection from vertical surfaces in porous media: variable surface temperature or heat flux. Int. J. Heat Mass Transfer 36: $1485-1493$. DOI: $10.1016 /$ S00179310(05)80059-6

Ingham, D.B., J.H. Merkin and I. Pop, 1982. Flow past a suddenly cooled vertical flat surface in a saturated porous medium. Int. J. Heat Mass Transfer, 25: 1916-1919. DOI: 10.1016/00179310(82)90117-X

Irwan, M.A.M., A.M. Fudhail, C.S.N. Azwadi and G. Masoud, 2010. Numerical investigation of incompressible fluid flow through porous media in a lid-driven square cavity. Am. J. Applied Sci., 7: 1341-1344. DOI: 10.3844/ajassp.2010.1341.1344

Johnson, C.H. and C. Ping, 1978. Possible similarity solutions for free convection boundary layers adjacent to flat plates in porous media. Int. J. Heat Mass Transf., 21: 709-718. DOI:10.1016/00179310(78)90032-7

Merkin, J.H., 1980. Mixed convection boundary layer flow on a vertical surface in a saturated porous medium. J. Eng. Math., 14: 301-313. DOI: 10.1007/BF00052913

Nield, D.A. and A. Bejan, 2006. Convection in Porous Media. 3rd Edn., Springer, New York, ISBN: 0387290966, pp: 640.
Nakayama, A. and C.D. Ebinuma, 1990. Transient nonDarcy forced convective heat transfer from a flat plate embedded in a fluid-saturated porous medium. Int. J. Heat Fluid Flow, 11: 249-253. DOI: 10.1016/0142-727X(90)90044-C

Raptis, A.A., 1983. Unsteady free convective flow through a porous medium. Int. J. Eng. Sci., 21: 345-348. DOI: 10.1016/0020-7225(83)90118-0

Shigeo, K., 1989. Transient forced and natural convection heat transfer about a vertical cylinder in a porous medium. Int. J. Heat Mass Transfer, 32: 617-620. DOI: 10.1016/0017-9310(89)90150-6

Takhar, H.S. and O.A. Beg, 1996. Non-Darcy effects on convective boundary layer flow past a semiinfinite vertical plate in saturated porous media. Heat Mass Trans., 32: 33-44. DOI: 10.1007/s002310050088

Telles, R.S. and O.V. Travisan, 1993. Dispersion in heat and mass transfer natural convection along vertical boundaries in porous media. Int. J. Heat Mass Transfer, 36: 1357-1365. DOI: 10.1016/S0017-9310(05)80103-6 